\title{
SISTEMA DE RECONOCIMIENTO DE GESTOS PARA CIRUGÍA ASISTIDA CON LA MANO
}

\author{
C. López-Casado, E. Bauzano, I. Rivas-Blanco, C.J. Pérez-del-Pulgar, and V.F. Muñoz \\ Departamento de Ingenierá de Sistemas y Automática, Universidad de Málaga, mclopezc@uma.es
}

\begin{abstract}
Resumen
Las técnicas de cirugía mínimamente invasiva están aumentando en cantidad y complejidad para cubrir una más amplia gama de intervenciones. Más específicamente, la Cirugía Laparoscópica Asistida con Mano (Hand-assisted laparoscopic surgery, HALS) implica el uso de una mano del cirujano dentro del paciente, mientras que la otra maneja una herramienta laparoscópica. En este caso, procedimientos quirúrgicos realizados con una herramienta adicional requieren la ayuda de un asistente. Además, en el caso de un asistente robótico, es obligatoria una comunicación fluida. Esta Interacción Hombre-Máquina debe combinar tanto órdenes explícitas como información implícita de los gestos quirúrgicos. En este contexto, este trabajo se centra en el desarrollo de un sistema de reconocimiento de gestos de la mano. Éste se basa en un modelo oculto de Markov (HMM) con un paso de entrenamiento automatizado mejorado. Este algoritmo también puede aprender durante el procedimiento quirúrgico en línea.
\end{abstract}

Palabras clave: aprendizaje colaborativo, robots asistivos, técnicas de interacción.

\section{INTRODUCCIÓN}

La Cirugía Mínimamente Invasiva se ha convertido en una de las técnicas quirúrgicas más importantes debido a su capacidad de reducir la convalecencia post-operatoria de los pacientes. Sin embargo, impone varias restricciones como la pérdida de la percepción de la profundidad visual, la sensación táctil limitada y el movimiento inverso de las herramientas laparoscópicas.

Estas técnicas han evolucionado a lo largo del tiempo para lograr dos objetivos: el bien del paciente o la reducción de las restricciones del cirujano mencionadas anteriormente. La Cirugía Laparoscópica Asistida con la Mano es un ejemplo de una variante de ésta. En ella se usa una de las manos del cirujano en el espacio de trabajo quirúrgico para mejorar la sensación táctil y la destreza, sin embargo, la incisión es mayor de lo habitual.
La introducción de asistentes robóticos como colaboradores en este tipo de técnicas [1] obtienen beneficios tanto para el paciente como mejorar los movimientos y la percepción de los cirujanos. Este tipo de sistemas robóticos deben tener una Interfaz Hombre-Máquina (HMI) intuitiva para que puedan ayudar al cirujano adecuadamente durante el procedimiento quirúrgico. Un campo de investigación relevante se centra en el reconocimiento de las tareas del cirujano. Este reconocimiento se basa normalmente en la detección de gestos de la herramienta [2], o gestos de la mano si se utiliza HALS (Hand-Assisted Laparoscopic Surgery). Esta última técnica requiere un mapeo de la anatomía de la mano del cirujano antes del reconocimiento en sí. Las metodologías de mapeo se clasifican en: métodos basados en mapas de profundidad (características volumétricas) y métodos basados en esqueletos (características de articulaciones y ángulos) [3]. Aunque las cámaras digitales son las fuentes más comunes de extracción de datos, otros trabajos prefieren el uso de acelerómetros de 3 ejes [4].

Las manos son el área anatómica más relevante para detectar los gestos del cirujano. La literatura presenta varios algoritmos para el Reconocimiento de Gestos de la Mano (HGR) [5] como los Modelos Ocultos de Markov (HMM) y el Dynamic Time Warping (DTW) entre otros. Los primeros se utilizan en trabajos como el reconocimiento automático del lenguaje de signos a través de un guante con acelerómetros [6] o el reconocimiento de patrones de señales de electromiografía [7]. Por otro lado, este último aparece en el reconocimiento en tiempo real de los gestos de las manos [8] o en la detección de patrones de comportamiento del Trastorno por Déficit de Atención e Hiperactividad [9]. Ambos algoritmos tienen sus propias ventajas e inconvenientes [10] pero su tasa de éxito de reconocimiento es muy similar.

Estos algoritmos de reconocimiento de gestos requieren métricas y métodos de agrupamiento para comparar y clasificar nuevos datos medidos con patrones de referencia. La detección de umbrales para el análisis comparativo se basa comúnmente en distancias euclídeas, Hamming [4] o en el uso 


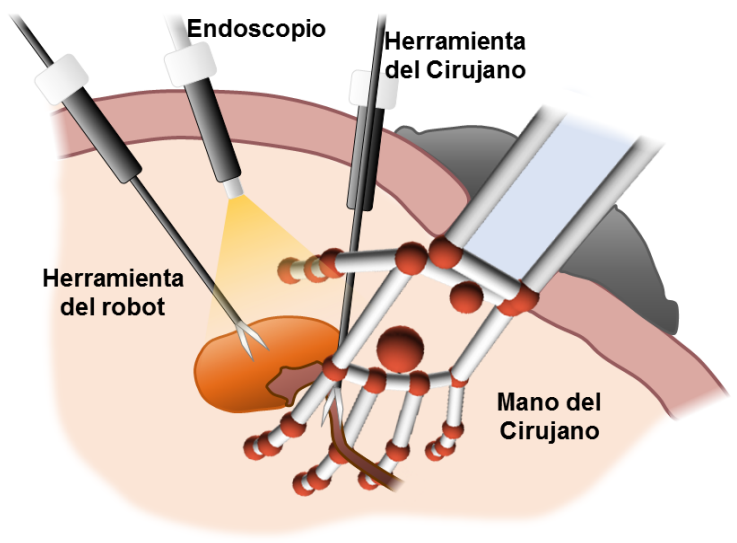

Figura 1: Espacio de trabajo.

más reciente de las distancias de Mahalanobis [11]. Además, la clasificación por clusters se realiza mediante técnicas como k-means [12] o el Gaussian Mixture Model (GMM) [13].

Este artículo propone un algoritmo HGR mejorado basado en técnicas HMM para ser utilizado dentro de una plataforma robótica para HALS. El núcleo de este algoritmo es la automatización del entrenamiento HMM, junto con una actualización en línea de la biblioteca de gestos, teniendo en cuenta el índice de confianza del gesto reconocido. El objetivo de esta plataforma es ayudar al cirujano como lo haría un asistente humano. Así, el reconocimiento de la etapa quirúrgica y la decisión de la acción dentro del flujo de trabajo quirúrgico son factores clave en esta plataforma.

Este artículo está organizado de la siguiente manera. La Sección 2 presenta el planteamiento del problema. La sección 3 profundiza en el proceso de adquisición de datos y entrenamiento necesario para el reconocimiento de gestos que se explica en la sección 4. Los resultados experimentales se muestran en la sección 5. Finalmente, las conclusiones se presentan en la sección 6.

\section{PLANTEAMIENTO DEL PROBLEMA}

La técnica HALS requiere la presencia de una mano del cirujano dentro del espacio de trabajo quirúrgico mientras que la otra maneja una herramienta laparoscópica. Esta configuración no permite el uso de una herramienta adicional, que suele ser útil para varios procedimientos laparoscópicos. Para minimizar esta restricción, este trabajo propone el uso de un asistente quirúrgico robótico de dos brazos. Un brazo orienta el endoscopio hacia la región de interés, y el otro maneja una herramienta robótica adicional (Fig. 1). Es-
Cuadro 1: Resección renal.

\begin{tabular}{|l|l|l|l|}
\hline Etapa & Actor & Herramienta & Descripción \\
\hline 1 & Cirujano & Mano & $\begin{array}{l}\text { Separación } \\
\text { de las ad- } \\
\text { herencias } \\
\text { intreperito- } \\
\text { neales }\end{array}$ \\
\hline 2 & Robot & Fórceps & $\begin{array}{l}\text { Sujetar un } \\
\text { punto del } \\
\text { uréter y } \\
\text { del vaso } \\
\text { sanguíneo }\end{array}$ \\
\hline 3 & Cirujano & Mano & $\begin{array}{l}\text { Sujetar punto } \\
\text { otro purcano al } \\
\text { cercano } \\
\text { anterior }\end{array}$ \\
\hline 4 & Cirujano & Clipper & $\begin{array}{l}\text { Añadir dos } \\
\text { clips entre } \\
\text { los puntos } \\
\text { sujetos }\end{array}$ \\
\hline 5 & Robot & Tijeras & $\begin{array}{l}\text { Cortar el } \\
\text { uréter y el } \\
\text { vaso san- } \\
\text { guíneo entre } \\
\text { los dos clips }\end{array}$ \\
\hline 6 & Cirujano & Mano & $\begin{array}{l}\text { Extraer } \\
\text { la zona } \\
\text { cortada }\end{array}$ \\
\hline & &
\end{tabular}

ta segunda herramienta permite realizar procedimientos laparoscópicos que requieren ambas herramientas (por ejemplo, la sutura) mediante movimientos automáticos de colaboración.

Las acciones del asistente robótico dependen principalmente de la tarea en curso del cirujano. Los movimientos realizados por la mano del cirujano son diferentes dependiendo de la etapa del procedimiento quirúrgico. Por lo tanto, el objetivo principal consiste en hacer que estos movimientos sean gestos comprensibles para el asistente robótico. Estos gestos pueden ser tanto tareas quirúrgicas como órdenes directas (por ejemplo, sujetar una gasa o apuntar con el dedo para enfocar la cámara a una posición específica).

El cuadro 1 muestra el protocolo HALS de una resección renal total adaptada al uso de un asistente robótico. Cada etapa de este protocolo puede describirse mediante el reconocimiento de una serie de gestos quirúrgicos realizados por la mano del cirujano:

Agarrar. Abrir y cerrar la mano. Útil para detectar el agarre de una gran masa de tejido o un órgano, aunque también se puede hacer para separar adherencias. 


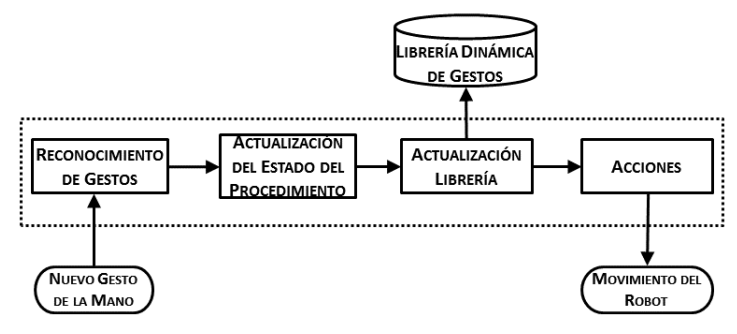

Figura 2: Flujo de trabajo general.

Tijeras. Dedos índice y medio extendidos, separar y unir sus puntas como un mecanismo de tijeras. Permite ordenar a la herramienta del robot que realice una acción con su herramienta.

Fórceps. Las yemas de los dedos pulgar e índice se acercan y retroceden como un fórceps. Detectar cuando la mano del cirujano está tirando de un tejido o vaso delgado.

La figura 2 muestra el flujo de trabajo de este protocolo. Cuando se detecta un nuevo gesto, el algoritmo de reconocimiento de gestos intenta reconocerlo. Una vez que se ha reconocido un nuevo gesto, se actualiza la etapa del protocolo HALS y se actualiza la biblioteca dinámica de gestos con la información del gesto recién reconocido. Finalmente, la acción a realizar por el robot quirúrgico para asistir adecuadamente al cirujano es comunicada y luego ejecutada por el robot.

\section{ENTRENAMIENTO DE GESTOS OFF-LINE}

El primer elemento del flujo de trabajo general del sistema introducido en la Fig. 2 consiste en el reconocimiento de gestos. Este algoritmo requiere el entrenamiento inicial de una librería dinámica de gestos. Por lo tanto, esta sección explica los pasos que sigue este trabajo para obtener el modelo requerido para cada gesto dentro de la biblioteca de gestos dinámicos.

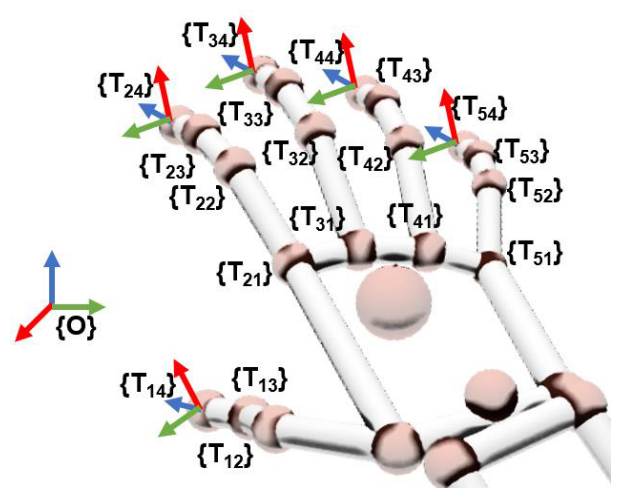

Figura 3: Ejes de coordinadas.
El proceso de entrenamiento con gestos ha sido diseñado de tal manera que sólo la información más relevante de un gesto se procesa automáticamente y se codifica en secuencias de etiquetas, tal y como se describe en la sección 3.1. Estos datos se procesan mediante el algoritmo de entrenamiento con gestos (sección 3.2), que se ha implementado mediante una red HMM, para obtener un conjunto de patrones para cada uno de los gestos propuestos.

\subsection{ADQUISICION DE DATOS Y PROCESADO}

El proceso de entrenamiento de gestos comienza con el movimiento de la mano del cirujano para realizar uno de los gestos explicados en la Sección 2. La adquisición de datos de la mano del cirujano puede realizarse mediante un guante inteligente. Este dispositivo mide la posición de cada una de las articulaciones $T_{i j}$ de los dedos del cirujano con respecto al dispositivo sensor O. (Fig. 3). En cada fotograma, el índice $i$ denota el dedo $(1=$ pulgar; $2=$ índice; $3=$ corazón; $4=$ anular; $5=$ meñique) y $j$ se refiere a las articulaciones entre sus huesos contiguos ( $1=$ metacarpo-proximal; 2 $=$ proximal-medio; $3=$ medio-distal; $4=$ yema del dedo). Todos los orígenes de coordenadas $T_{i j}$ están orientados de tal manera que sus vectores $z_{i j}$ tienen la dirección de la falange $j$ relacionada con el dedo $i$. Éstos pueden redefinirse como un conjunto de parámetros geométricos (las llamadas características) como la flexión de las articulaciones $q_{i j}$, la distancia $l_{i}$ y la orientación $\beta_{i}$ entre las puntas de los dedos contiguos:

$$
\begin{aligned}
l_{i} & =\overline{T_{i 4} T i+14} \\
\beta_{i} & =\operatorname{atan} 2\left(\left\|z_{i 4} \times z_{i+14}\right\|, z_{i} \cdot z_{i+14}\right) \\
q_{i j} & =\operatorname{atan} 2\left(\left\|z_{i j} \times z_{i j+1}\right\|, z_{i j} \cdot z_{i j+1}\right) \\
f_{g} & =<l_{1} \cdots l_{4} \beta_{1} \cdots \beta_{4} q_{12} \cdots q_{53}>=<f_{x}>
\end{aligned}
$$

En (1), todas las características de un gesto $g$ específico se reúnen como un componente $f_{x}$ dentro de un vector de característica $f_{g}$. Esta nueva definición de la postura de la mano completa en términos de características simplifica el flujo de trabajo del entrenamiento que aparece en la Fig. 4. En primer lugar, el elemento de adquisición de características graba $\mathrm{N}$ veces un gesto específico g. Cada repetición $n$ almacena una secuencia de características con la trayectoria completa de todos los vectores característicos $f_{g, n}[k]$ discretizados con $K_{n}$ muestras en intervalos de tiempo de $\Delta t$. Por lo tanto, el conjunto de todas las secuencias de características $F_{g}$ es:

$$
F_{g}=\left\{f_{g, n}[k], n \in 1 \ldots N, k \in 0 \ldots K_{n}\right\} ; t_{k}=k \Delta t
$$

Tanto la trayectoria como la velocidad de la mano pueden cambiar para cada repetición $n$. Así, el 
número de muestras $K_{n}$ adquiridas puede ser diferente también. Estos datos en bruto $F_{g}$ deben procesarse en los siguientes pasos del flujo de trabajo mostrado en la Fig. 4 para eventualmente obtener la secuencia codificada para una red HMM:

1. Ajuste de características. Cada secuencia de características $f_{g, n}$ de todas las $N$ repeticiones deben ser comparables en muestreo, tiempo y tamaño. El algoritmo Dynamic Time Warping (DTW) se puede utilizar para encontrar una alineación óptima entre dichas secuencias de características. Más específicamente, DTW corrige el retardo, iguala el número de muestras $K_{n}=K$ y proporciona un valor cuantitativo de la similitud $\sigma_{n m}$ entre dos secuencias de características diferentes $f_{g, i}$ y $f_{g, j}$, siendo $i, j \in 1 \ldots .$. . De esta manera, cuanto más cerca esté $\sigma_{n m}$ de 0 , más similares son ambas secuencias. DTW se aplica en este paso a eliminar cualquier repetición que esté etiquetada como no similar debido a errores en las mediciones de los sensores. Este proceso eventualmente lleva a una cantidad reducida de $M<N$ secuencias de características válidas $W_{g}=D T W\left(F_{g}\right)$ con longitud fija de muestras $K$.

2. Selección de características. El entrenamiento de un gesto específico por lo general requiere sólo un subconjunto de características $S_{g} \subseteq W_{g}$ que defina con precisión el significado de los movimientos de las manos del cirujano. Por ejemplo, el gesto de la tijera se realiza mediante la separación del índice y las puntas de los dedos del medio, por lo que las características más relevantes son $l_{2}, \beta_{2}$. De esta manera se ha implementado un método de selección de características para elegir automáticamente estas características más relevantes basándose en la siguiendo criterios:

Similitud. Para cada componente de la característica $f_{x}$, DTW es aplicado con respecto a las otras características del vector $f_{g}$. Si dos o más componentes $f_{x}$ son similares, entonces sólo se usa uno para el entrenando del gesto. Como antes, esta comparación se hace en términos de la distancia euclidiana $\sigma_{n m}$ dada por el algoritmo DTW.

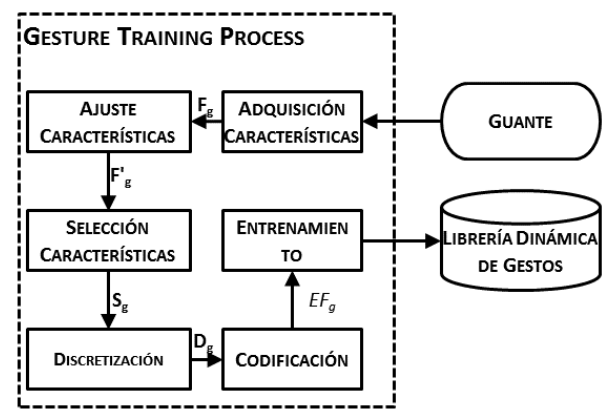

Figura 4: Flujo de trabajo del entrenamiento.
Relevancia. Secuencias de características con picos máximos por debajo de un umbral específico son obviadas. En otras palabras, las características con movimientos no relevantes son ignorados para el entrenamiento. Experimentalmente, los valores usuales de tales umbrales se sitúan en torno a los siguientes valores: $1 \mathrm{~cm}$ para las características de distancia $l_{i}$ y $15^{\circ} \mathrm{C}$ para las características de ángulo $\beta_{i}, q_{i j}$.

3. Discretización. Las secuencias de características seleccionadas $S_{g}$ son procesadas por un método de discretización, que encuentra la cantidad óptima de clusters $c_{x}$ y almacena sus ubicaciones en un vector de valores centrales $C_{g}=\left(c_{1}, c_{2} \ldots c_{x}\right)$. El algoritmo elegido para esta tarea es el X-means [14], una variante de K-means que calcula el número óptimo de clusters mediante un criterio específico como la evaluación de Calinski-Harabasz. Estos valores centrales $C_{g}$ se utilizan para discretizar las características seleccionadas $S_{g}$ en $D_{g}$ mediante la distancia euclídea de cada muestra de característica con el valor central de componente. Además, se considera una zona de histéresis cercana a cada valor central para evitar valores máximos y minimizar las oscilaciones inestables debidas a los efectos del ruido. Los valores finales de cada componente de característica discreta $d_{x}[k]$ son enteros entre 0 y su número de valores centrales $\left(d_{x}[k] \in 0 \ldots \operatorname{dim}\left(C_{g}\right)-1\right)$.

4. Codificación. Todas las secuencias discretizadas $D_{g}$ de cada característica se combinan en una única secuencia codificada de etiquetas $E_{g}$.La secuencia etiquetada $e_{g, n}[k]$ es codificada mediante los componentes de la característica discretizada $d_{x}[k]$ y los componentes $c_{l}$ de los valores centrales $C_{g}(9)$ :

$$
e_{g, n}[k]=1+d_{1}[k]+\sum_{m=2}^{x}\left(\prod_{l=1}^{m-1} c_{l}\right) d_{m}[k]
$$

Esta expresión asigna una etiqueta única para cada combinación de todas las características.

\subsection{ENTRENAMIENTO DE PATRONES}

El conjunto codificado $E_{g}$ obtenido de cada repetición de la secuencias de características discretizadas $D_{g}$ se envía secuencialmente al proceso de entrenamiento (Fig. 4). El proceso de entrenamiento construye una red HMM asociada a cada uno de los gestos explicados en la Sección 2 mediante el algoritmo Baum-Welch. Cada una de estas redes consiste en un conjunto de gestos $\lambda_{g}$ con los siguientes parámetros entrenados:

$$
\lambda_{g}=\{S Q, S E, \mathbf{U}, \mathbf{V}, \pi\}
$$

En esta expresión, $S Q$ denota los estados del 
HMM, SE es el conjunto de todas las combinaciones posibles de las secuencias codificadas $E_{g}$ para cada muestra $e_{g, n}[k]$, la matriz de transición $\mathbf{U}$ es la distribución de probabilidad que indica las relaciones entre los estados, la matriz de emisión $\mathbf{V}$ es la distribución de probabilidad que establece el valor más probable de la secuencia codificada en cada estado, y $\pi$ es la distribución de estados inicial. Más concretamente, se ha elegido 5 estados $S Q$ para todos $\lambda_{g}$ entrenamientos. El conjunto de secuencias codificadas $S E$ incluye todas las combinaciones de $e_{g, n}$ para cada muestra $k$. $\mathbf{U}$ es inicialmente una matriz simétrica diagonal $5 \times 5$ donde las diagonales superior e inferior no son nulas, y la suma por filas (columnas) es igual a 1. Del mismo modo, $\mathbf{V}$ es inicialmente una matriz $5 \mathrm{x}\left(c_{1} c_{2} \cdots c_{x}\right)$ donde la suma por fila es igual a 1 .

Después de las $N$ repeticiones realizadas para cada uno de los gestos $g$ introducidos en la sección II, los conjuntos de gestos $\lambda_{g}$ resultantes se almacenan en la biblioteca dinámica de gestos. Estos datos se utilizan en el proceso de reconocimiento de gestos, que se explica con más detalle en la siguiente sección.

\section{PROCESO DE RECONOCIMIENTO DE GESTOS}

La biblioteca dinámica de gestos que se utiliza durante el proceso de reconocimiento está compuesta por los registros de gestos que se realizaron durante el proceso de entrenamiento fuera de línea. Con el fin de incluir nuevos registros realizados durante la cirugía en sí, se ha diseñado un procedimiento en línea para incluirlos. De esta manera, la Biblioteca Dinámica de Gestos permite adaptarse a los cambios que el cirujano puede realizar sobre los gestos entrenados y, en consecuencia, mejorar el rendimiento global del proceso de reconocimiento. A continuación se detallará todo el proceso desde el reconocimiento del gesto hasta la actualización de la librería (Fig. 5).

Una vez que se ha grabado y codificado un nuevo registro, se inicia el proceso de reconocimiento. La secuencia codificada en línea, $E$, se procesa mediante un algoritmo Forward-Backward para cada conjunto de gestos $\lambda_{g}$ de datos entrenados. Dicho algoritmo calcula las probabilidades $P_{g}$ convertidas en una escala logarítmica y, como resultado, devuelve el gesto $g$ que ha obtenido la mayor probabilidad $P_{\max }$. Asimismo, se obtiene un valor de Índice de Confianza (IC) entre 0 (confianza nula) y 1 (confianza total). Este índice se calcula mediante las dos probabilidades más altas, la del gesto reconocido, $P_{\max }$ y la del gesto reconocido en segunda

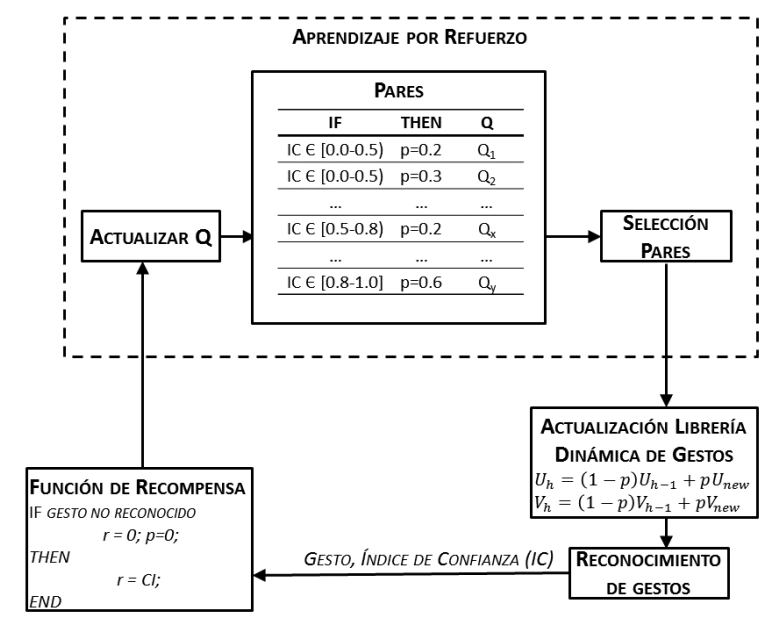

Figura 5: Procedimiento de aprendizaje en línea.

posición, $P_{2}$ :

$$
I C=\min \left(\frac{P_{2}-P_{\max }}{2 P_{\max }}, 1\right)
$$

La forma en que la Biblioteca de gestos dinámicos se actualiza con la información del registro que acaba de ser reconocido es a través de las matrices de transición (U) y de probabilidad de emisión $(\mathbf{V})$. Cada vez que hay que reconocer un nuevo registro, $E$, se calculan las matrices de probabilidad de transición y de emisión de esta nueva secuencia. Éstas se utilizarán para obtener las nuevas matrices del conjunto de gestos $\lambda_{g}(9)$ que acaba de ser reconocido, utilizando (12) y (13):

$$
\begin{aligned}
& \mathbf{U}_{h}=(1-p) \mathbf{U}_{h-1}+p \mathbf{U}_{\text {new }} \\
& \mathbf{V}_{h}=(1-p) \mathbf{V}_{h-1}+p \mathbf{V}_{\text {new }}
\end{aligned}
$$

donde $\mathbf{U}_{h}$ es la nueva matriz de probabilidad de transición del conjunto de gestos $\lambda_{g} ; \mathbf{U}_{h-1}$ es la matriz de probabilidad de transición anterior del conjunto de gestos $\lambda_{g}$ y $\mathbf{U}_{\text {new }}$ es la matriz de probabilidad de transición del nuevo registro. Del mismo modo, $\mathbf{V}_{h}$ es la nueva matriz de probabilidad de emisión del conjunto de gestos $\lambda_{g} ; \mathbf{V}_{h-1}$ es la anterior y $V_{\text {new }}$ es la matriz de probabilidad de emisión del nuevo registro. Finalmente, p es el valor utilizado para ponderar el nuevo registro con respecto a los antiguos. Este parámetro se elegirá de forma que se maximice el IC, como se explica a continuación.

Cuando el nuevo registro ha sido mal reconocido, el cirujano informa al sistema a través de la interfaz HMI, que el valor de p es cero, es decir, este registro no se utiliza para actualizar la Biblioteca Dinámica de Gestos. Por el contrario, cuando el sistema reconoce correctamente el registro, se debe elegir p para mejorar el proceso global. Para evitar un sistema estático donde los pesos, p, son definidos y fijados durante el proceso de diseño, se ha utilizado un algoritmo de aprendizaje de re- 
fuerzo (RL) para hacer que el sistema aprenda este parámetro. Esta técnica de aprendizaje trata de maximizar una recompensa que se recibe cuando el sistema toma una decisión. Dentro de este trabajo, el objetivo es maximizar el IC previamente definido, de manera que aumente el rendimiento general del proceso de reconocimiento. Así pues, la señal de recompensa del algoritmo RL será el índice de confianza definido en (5).

Por otro lado, la decisión a tomar es qué valor de peso, p, se utilizará para actualizar la Biblioteca Dinámica de Gestos. Como esta decisión depende también del valor del IC, el algoritmo RL se ha organizado para tomar decisiones independientes en función del nivel de confianza en el reconocimiento. Por lo tanto, los registros con IC alto tenderán a valores de ponderación más altos que los reconocidos con IC extremadamente baja. Aunque el IC puede tomar valores entre 0 y 1 , se ha dividido en 3 grupos. De esta manera, sólo se aprenden tres valores de peso, $\mathrm{p}$, uno por grupo de IC. IC $=[0.0-$ 0.5),[0.5-0.8),[0.8-1.0]. Como se puede observar, el tamaño de cada grupo disminuye a medida que aumenta el valor de IC, lo que hace que sólo los mejores reconocimientos (IC más alto) tengan un alto impacto en la actualización de la Biblioteca Dinámica de Gestos.

Por otro lado, los valores de peso, $\mathrm{p}$, han sido discretizados teniendo en cuenta las siguientes consideraciones: (1) las nuevas matrices registradas no pueden sustituir completamente a las anteriores porque se perdería todo el histórico; por lo tanto, $\mathrm{p}=1$ se descarta. (2) las nuevas matrices de registro no pueden rechazarse si el gesto se ha detectado correctamente, por lo que se descarta $\mathrm{p}=0$. $\mathrm{Y}$ (3), el conjunto de gestos tiene que incluir mucha información de varias fuentes, es decir, diferentes registros de gestos. Para lograrlo, se prefiere que los valores de $\mathrm{p}$ no sean demasiado altos porque los valores más altos implican la pérdida de información de los registros anteriores. En consecuencia, los posibles valores de $\mathrm{p}$ se han discretizado en: $\mathrm{p}$ $=0.2,0.3,0.4,0.5,0.6$.

De acuerdo con la discretización y agrupación que se ha realizado, el algoritmo RL tiene diferentes valores de peso a elegir por grupo de IC. Es decir, para cada valor de CI dentro de un grupo, el algoritmo RL tiene cinco valores $\mathrm{p}$ diferentes para elegir (Tabla PARES en Fig. 5).

Para poder tomar la decisión, es decir, elegir un valor p dentro de los 5 posibles dentro de un grupo de IC, se ponderan estas reglas (grupo de IC - p). Cada par está asociado a un valor Q que se utilizará para tomar la decisión. El valor Q se actualiza cuando se recibe la recompensa según (algoritmo SARSA):

$$
\delta_{h}=\alpha\left(r_{h+1}+\gamma Q_{h+1}-Q_{h}\right)
$$

Donde $Q_{h+1}$ es el valor $Q$ en la iteración $h+i$; $r_{h+1}$ es la recompensa en esta iteración, es decir, el valor IC; $\alpha$ es la tasa de aprendizaje y $\gamma$ es la tasa de descuento. El ritmo de aprendizaje determina la importancia del nuevo conocimiento sobre la información antigua, mientras que el factor de descuento determina la importancia de las recompensas futuras.

Como la técnica de RL se basa en descubrir qué acciones son las más recompensadas probándolas, este tipo de algoritmo conlleva un término medio entre la exploración y la explotación: el sistema tiene que explotar lo que ya sabe, pero también tiene que explorar nuevas acciones. Para determinar cómo se seleccionan las reglas en base a su valor $\mathrm{Q}$, se ha utilizado la estrategia de exploración $\varepsilon-$ greedy. Esta estrategia selecciona aleatoriamente un par (grupo CI - p) con probabilidad $\varepsilon$, mientras que el par con el valor $\mathrm{Q}$ más alto se selecciona con probabilidad $1-\varepsilon$.

\section{RESULTADOS}

El objetivo del experimento es analizar el rendimiento general del Proceso de Reconocimiento de Gestos que se ha detallado en este artículo. La comparación se realiza entre tres configuraciones diferentes del algoritmo: sólo usando el Proceso de Reconocimiento de Gestos sin actualización de la Biblioteca Dinámicas de Gestos; usando el Proceso de Reconocimiento de Gestos junto con la actualización en línea de la Biblioteca explicado en la sección 4 pero con un peso fijo $(\mathrm{p}=0.5)$, es decir, sin algoritmo RL para inferir los valores de peso; y finalmente, todo el proceso incluyendo el enfoque RL. El proceso de entrenamiento ha consistido en 30 repeticiones de los tres gestos de las manos que se explican en la sección 2.

El algoritmo RL ha sido configurado usando una tasa de descuento $(\gamma)$ de 0.9 y una tasa de aprendizaje $(\alpha)$ de 0.3 , lo que hace que el sistema considere la información pasada pero también la nueva. En cuanto al uso de nuevos conocimientos para explorar, se ha configurado $\varepsilon$ con un valor inicial de 0.4 en los primeros 20 gestos para permitir la exploración de nuevos valores de peso y luego se ha reducido a 0.2 para obtener un mayor aprovechamiento de los valores ya conocidos y permitir así la convergencia del factor de peso.

La Biblioteca Dinámica de Gestos ha sido utilizada para realizar tres diferentes realizaciones de las tres configuraciones ya explicadas. Cada realización incluía la detección de 60 gestos. Junto con el tipo de gesto detectado, el proceso obtiene el índice de confianza que informa sobre la fiabilidad de 
la detección. En el cuadro 2 se muestra una comparación del porcentaje de reconocimientos con un índice de confianza superior a 0,5. Como se observa, el porcentaje de detección con una confianza superior a 0,5 aumenta al incluir el proceso en línea, siendo máximo al utilizar el algoritmo RL para inferir la ponderación que se utilizará para actualizar la librería con el nuevo gesto.

\section{Cuadro 2: $\% I C>0,5$}

\begin{tabular}{|l|l|l|l|}
\hline & $\begin{array}{l}\text { Sólo } \\
\text { Recono- } \\
\text { cimiento }\end{array}$ & $\begin{array}{l}\text { Recono- } \\
\text { cimiento } \\
+\mathbf{p = 0 . 5}\end{array}$ & $\begin{array}{l}\text { Recono- } \\
\text { cimiento } \\
+ \text { RL }\end{array}$ \\
\hline Exp.1 & $36.667 \%$ & $58.33 \%$ & $76.667 \%$ \\
\hline Exp.2 & $21.667 \%$ & $63.33 \%$ & $75 \%$ \\
\hline Exp.3 & $35 \%$ & $66.667 \%$ & $75 \%$ \\
\hline
\end{tabular}

Un análisis más profundo de los resultados del experimento muestra que el número de reconocimientos con plena confianza $(\mathrm{IC}=1,0)$ en los resultados también aumenta cuando se utiliza el algoritmo RL (Cuadro 3). Este resultado, junto con el anterior, hace que los falsos positivos globales se reduzcan cuando se utiliza el algoritmo RL. Los resultados anteriores revelan que la contribución del proceso de VR en línea aumenta el rendimiento del proceso de reconocimiento.

\begin{tabular}{|l|l|l|l|}
\multicolumn{5}{c}{ Cuadro 3: \%IC=1,0 } \\
\hline & $\begin{array}{l}\text { Sólo } \\
\text { Recono- } \\
\text { cimiento }\end{array}$ & $\begin{array}{l}\text { Recono- } \\
\text { cimiento } \\
+\mathbf{p = 0 . 5}\end{array}$ & $\begin{array}{l}\text { Recono- } \\
\text { cimiento } \\
+ \text { RL }\end{array}$ \\
\hline Exp.1 & $21.667 \%$ & $45 \%$ & $60 \%$ \\
\hline Exp.2 & $6.667 \%$ & $46.667 \%$ & $60 \%$ \\
\hline Exp.3 & $20.277 \%$ & $50 \%$ & $61.667 \%$ \\
\hline
\end{tabular}

Como se explicó en la Sección IV, se aprenden tres factores de peso diferentes, dependiendo de la agrupación del IC. La Fig. 6 muestra la evolución del factor de ponderación, p, en una de las tres realizaciones. Los demás se comportan de la misma manera.

Como se puede ver en la Fig. 6a, al inicio del pro-

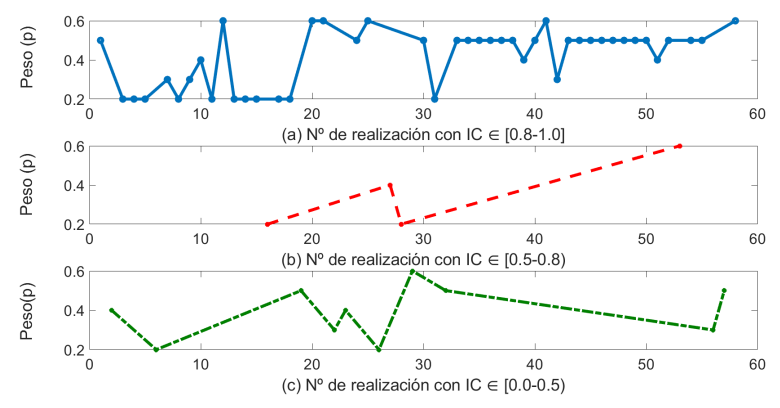

Figura 6: Aprendizaje de la ponderación.
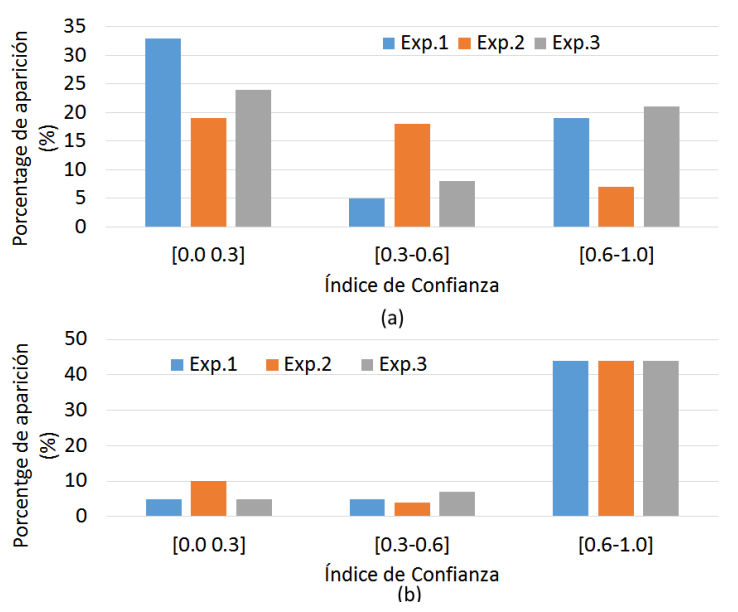

Figura 7: Porcentage de aparición del IC (a) Sin aprendizaje en línea. (b) Con aprendizaje RL.

ceso de aprendizaje hay una alta variabilidad en el valor del peso, pero debido a la realimentación de la recompensa termina convergiendo a $p=0.5$. Este valor es uno de los más altos posibles, lo que hace que la contribución del nuevo gesto a la biblioteca sea alta también cuando el índice de confianza es alto. En las agrupaciones restante del IC, no ha habido suficientes muestras para hacer converger el algoritmo como se muestra en las Fig. $6 \mathrm{~b}$ y Fig. 6c. El número de muestras cuando $\mathrm{IC}$ es inferior a 0,6 se reduce significativamente cuando se utiliza el algoritmo RL, como muestra la Fig. 7. Estos gráficos muestran cómo ha cambiado completamente la tendencia. Cuando no se utiliza ningún algoritmo RL, el rango más poblado es el[0.0-0.5], pero cuando se utiliza este algoritmo, el rango más grande es el opuesto, [0.8-1.0].

A través de estos resultados se afirma que el uso del proceso de RL en línea mejora el rendimiento general del proceso de reconocimiento. La confianza en el reconocimiento se incrementa por encima del $30 \%$ en relación con el uso exclusivo del proceso UPR. La mayor cantidad de gestos reconocidos cambia de tener un índice de confianza bajo a un índice de confianza alto, haciendo que el sistema sea más confiable para ser usado en una arquitectura cognitiva HALS, como la que ha sido implementada y probada.

\section{CONCLUSIONES}

Este trabajo ha presentado un HGR mejorado para mejorar la comunicación entre el asistente y el cirujano para un escenario de colaboración en HALS. Este HGR se compone de dos elementos principales. En primer lugar, el proceso de entrenamiento se dedica a reconocer los gestos a partir de un patrón de características establecido por 
medio de redes HMM que se entrenan para cada uno de los gestos y se guardan en una biblioteca dinámica de gestos. En segundo lugar, el reentrenamiento en línea de cada uno de los gestos añadiendo el último gesto reconocido en caso de que coincida con alguien de la Biblioteca de Gestos Dinámicos con cierta confianza. Los resultados experimentales de este sistema han demostrado cómo el proceso en línea de RL hace que el sistema mejore su rendimiento general, aumentando la confianza en cada gesto de reconocimiento.

\section{English summary}

\section{Cognitive Gesture Recognition Ap- proach for a Surgical Robot Assistant}

\section{Abstract}

Minimally Invasive Surgery techniques are growing in quantity and complexity to cover a wider range of interventions. More specifically, the Hand Assisted Laparoscopic Surgery (HALS) involves the use of one surgeon's hand inside the patient whereas the other one manages a single laparoscopic tool. In this scenario, those surgical procedures performed with an additional tool require the aid of an assistant. Furthermore, in the case of a human-robot assistant a fluid communication is mandatory. This Human-Machine Interaction must combine both, explicit orders and implicit information from the surgical gestures. In this context, this paper focuses on the development of a hand gesture recognition system for $H A L S$. The recognition is based on a Hidden Markov Model (HMM) algorithm with an improved automated training step, which can also learn during the online surgical procedure.

Keywords: Collaborative learning, Assistive robots, Interaction techniques.

\section{Referencias}

[1] L. Zorn, F. Nageotte, et al., A Novel Telemanipulated Robotic Assistant for Surgical Endoscopy: Preclinical Application to ESD, IEEE Transactions on Biomedical Engineering 65 (4) (2018) 797-808.
[2] E. Bauzano, B. Estebanez, et al., Collaborative Human-Robot System for HALS Suture Procedures, IEEE Systems Journal 10 (3) (2016) 957-966.

[3] T. D'Orazio, R. Marani, et al., Recent trends in gesture recognition: how depth data has improved classical approaches, Image and Vision Computing 52 (2016) 56-72.

[4] R. Xie, X. Sun, et al., Similarity MatchingBased Extensible Hand Gesture Recognition, IEEE Sensors Journal 15 (6) (2015) 34753483.

[5] P. K. Pisharady, M. Saerbeck, Recent methods and databases in vision-based hand gesture recognition: A review, Computer Vision and Image Understanding 141 (2015) 152165.

[6] J. Galka, M. Masior, et al., Inertial Motion Sensing Glove for Sign Language Gesture Acquisition and Recognition, IEEE Sensors Journal 16 (16) (2016) 6310-6316.

[7] M. Rossi, S. Benatti, et al., Hybrid EMG classifier based on HMM and SVM for hand gesture recognition in prosthetics, in: 2015 IEEE International Conference on Industrial Technology (ICIT), IEEE, 1700-1705, 2015.

[8] G. Plouffe, A.-M. Cretu, Static and Dynamic Hand Gesture Recognition in Depth Data Using Dynamic Time Warping, IEEE Transactions on Instrumentation and Measurement 65 (2) (2016) 305-316.

[9] M. A. Bautista, A. Hernandez-Vela, et al., A Gesture Recognition System for Detecting Behavioral Patterns of ADHD, IEEE Transactions on Cybernetics 46 (1) (2016) 136-147.

[10] J. Raheja, M. Minhas, et al., Robust gesture recognition using Kinect: A comparison between DTW and HMM, Optik - International Journal for Light and Electron Optics 126 (11-12) (2015) 1098-1104.

[11] N. H. Duong, H. Dang Hai, A semi-supervised model for network traffic anomaly detection, in: 2015 17th International Conference on Advanced Communication Technology (ICACT), IEEE, 70-75, 2015.

[12] M. F. Lima, B. B. Zarpelao, et al., Anomaly detection using baseline and K-means clustering, in: SoftCOM 2010, 18th International Conference on Software, Telecommunications and Computer Networks, 305-309, 2010.

[13] Y. Song, Y. Gu, et al., A Kinect based gesture recognition algorithm using GMM and HMM, in: 2013 6th International Conference 
on Biomedical Engineering and Informatics, IEEE, 750-754, 2013.

[14] D. Pelleg, D. Pelleg, A. Moore, X-means: Extending K-means with Efficient Estimation of the Number of Clusters, in: THE 17TH INTERNATIONAL CONF. ON MACHINE LEARNING, 727-734, 2000.

CC (\$) $\underset{\text { Submitted }}{2018 \text { by the authors. }}$ (C) NC open access publication under the terms and conditions of the Creative Commons Attribution CC-BY-NC 3.0 license (http://creativecommons.org/licenses/by-nc/3.0/). 